\title{
Reflexiones sobre los estudios de función pulmonar en nuestra práctica clínica
}

Los estudios de función pulmonar pueden evaluar en forma objetiva y cuantificable los diferentes componentes de la función respiratoria: ventilación, difusión, perfusión, relación ventilación/perfusión, transporte gaseoso, mecánica de la respiración y control de la ventilación ${ }^{1,2}$.

Es importante hacer notar, que estas pruebas no permiten realizar diagnóstico etiológico, sino que informan el modo en que la patología o las condiciones ambientales alteran la fisiología normal. Son muy útiles para objetivar la gravedad de un trastorno funcional, establecer la necesidad de tratamiento o la respuesta al mismo, determinar el origen de la disnea, tos o sibilancias, evaluar el riesgo de exposición laboral y en el período preoperatorio evaluar el riesgo anestésico y/ o quirúrgicol.

El examen básico de medición de la función pulmonar es la espirometría ${ }^{1,2}$, que evalúa tanto la mecánica respiratoria, proceso por el cual los músculos respiratorios mueven a la pared torácica y al pulmón venciendo diferentes resistencias elásticas y de flujo, como también la ventilación, mecanismo a través del cual el aire inspirado llega a los alvéolos y el aire alveolar llega a la atmósfera, y que está directamente relacionado con la indemnidad de las vías aéreas. Las alteraciones de la mecánica respiratoria generan limitaciones de tipo restrictivo y las anomalías de las vías aéreas originan limitaciones de tipo obstructivo. Pudiendo coexistir ambas limitaciones en varias patologías. Este examen es de bajo costo, y puede ser realizado por cualquier profesional del equipo de salud, siempre que haya adquirido un entrenamiento apropiado y mantenga las habilidades adquiridas. La capacitación del personal que realiza los exámenes y la acreditación de los laboratorios de función pulmonar es una responsabilidad que debería recaer en especialistas de enfermedades respiratorias y en miembros de nuestra Sociedad.

La espirometría debería constituirse en un estudio de rutina en todas las personas con riesgo de padecer enfermedades respiratorias, en los enfermos respiratorios y en un examen primordial para pacientes que serán sometidos a cirugía torácica o extratorácica, considerándolo como un índice de evaluación del riesgo quirúrgico, y como pauta del manejo postoperatorio y de las técnicas quirúrgicas y anestésicas adecuadas a seguir ${ }^{1,2}$.

En los últimos años, relacionado con el aumento de la prevalencia de la Enfermedad Pulmonar Obstructiva Crónica (EPOC) asociada al tabaquismo, se han publicado numerosas guías de manejo de la EPOC nacionales e internacionales. Entre ellas destacan las recomendaciones de GOLD', que proponen la evaluación periódica de la función pulmonar o espirometría anual en pacientes con riesgo de desarrollar EPOC, como son las personas fumadoras mayores de 40 años. El estudio PLATINO (Proyecto latinoamericano de investigación en obstrucción pulmonar), cuyo objetivo principal fue medir la prevalencia de la EPOC en cinco centros de América Latina, utilizó la espirometría con este fin. En nuestro país estudió a 1.172 personas mayores de 39 años de la población general de Santiago demostrando una prevalencia de 16,9\% y un subdiagnóstico de EPOC de $14,8 \%$, definido como personas en las que se encontró una relación $V E F_{1} / C V F$ postbroncodilatador menor de 0,7 sin diagnóstico previo de la enfermedad. Además, se sabe que en adultos hay una pérdida fisiológica de $V E F_{1}$ de $30 \mathrm{ml} / a \tilde{n} o$, por lo tanto encontrar caídas superiores a esta cifra en fumadores asintomáticos, permitiría pesquisar precozmente personas susceptibles de padecer la enfermedad, aunque tengan una espirometría normal.

La Sociedad Chilena de Enfermedades Respiratorias (SER) a través de su Comisión de Función Pulmonar en el adulto, ha publicado recientemente un manual de procedimientos para que logremos a nivel nacional, mejorar y uniformar la ejecución de la espirometría ${ }^{4}$. Además, las Comisiones de Función Pulmonar en Adultos y en Pediatría organizaron conjuntamente en Julio del presente año, las $16^{a}$ Jornadas de Invierno cuyo objetivo ha sido dar a conocer el "estado del arte" de las pruebas de función pulmonar tanto en adultos como niños ${ }^{1}$. Para lograr este propósito contamos 
con la destacada participación de especialistas nacionales e invitados extranjeros de reconocido prestigio internacional como son los Drs. Andrew Colin, Josep Roca y Juan Carlos Vásquez.

En general el futuro de algunas de estas pruebas, como por ejemplo la medición de la capacidad de difusión con monóxido de carbono $\left(D_{L C O}\right)$, apunta a simplificarlas generando equipos de menor costo y tecnología más amigable, para poder realizarlas en forma masiva a nivel de atención primaria, como ha ocurrido con la espirometríal.

En nuestro país, el Ministerio de Salud, en relación con la incorporación del Asma infantil y de la EPOC, como patologías del plan de salud AUGE ("acceso universal y garantías explícitas"), ha dotado de espirómetros a la atención primaria y secundaria a lo largo de todo el país. Esto nos da una тиy buena oportunidad y un deber, como especialistas en enfermedades respiratorias, tanto de adultos como niños, de velar por lograr a nivel nacional la estandarización de la espirometría a nivel primario y secundario y aspirar a tener a nivel de atención secundaria laboratorios de función pulmonar que cuenten con equipamiento tecnológico y humano, que permita desarrollar además las otras pruebas básicas de exploración de la función pulmonar. Entre estas pruebas básicas se cuentan: la medición de volúmenes pulmonares, la ya citada $D_{L C O}$, la gasometría arterial, las pruebas de provocación bronquial y la medición de la capacidad física, con la prueba de caminata de 6 minutos y la prueba cardiopulmonar de ejercicio.

También debiéramos hacer esfuerzos por incorporar los conceptos de Control de Calidad, enunciados por el Dr. Juan Carlos Vásquez, en la rutina del quehacer de nuestros laboratorios de función pulmonar. Cada uno de estos laboratorios debiera mantener al día los siguientes documentos: a) manual de instalación de equipos, b) manual de procedimientos, c) manual técnico, d) base de datos, con todas las maniobras o al menos las tres mejores; e) base de datos con calibraciones o bitácora de calibraciones; e) gráficas de controles biológicos, f) bitácoras de problemas y acciones, g) bitácoras de mantenimientos, y h) registros de calidad de técnicas incluyendo porcentaje de pruebas que cumplen los criterios de ATS.

Finalmente, una tarea que aún está pendiente es la relacionada con contar con valores nacionales de referencia espirométrica ${ }^{5-8}$. Afortunadamente, en nuestro medio se han hecho avances significativos. Es así como la Dra. Solange Caussade, en representación del grupo pediátrico de la Pontificia Universidad Católica de Chile presentó en las $16^{a}$ Jornadas de Invierno, resultados preliminares de un estudio para obtener valores de referencia en niños. Han estudiado hasta la fecha 1.416 niños sanos de 6 a 19 años de ambos sexos de 6 comunas de la Región Metropolitana ${ }^{\text {. }}$ Por otra parte, en adultos también estamos haciendo grandes esfuerzos, a nivel de la Comisión de Función Pulmonar de la SER, para establecer nuevas ecuaciones nacionales ${ }^{9-11}$. Para ello se ha aumentado el número de espirometrías en adultos mayores sanos incorporadas a las muestras poblacionales para obtener los valores de referencia ${ }^{l}$, ya que se ha demostrado que es imperioso reemplazar los valores de referencia de Knudson y colaboradores ${ }^{7}$, que actualmente están en uso, por valores de referencia nacionales que den más cabal cuenta de la realidad de nuestra población en cuanto a los valores espirométricos ${ }^{13-15}$, que son de tanta trascendencia para tomar decisiones médicas, quirúrgicas, sociales y laborales.

Dra. Mónica Gutiérrez C. Coordinadora, Comisión Función Pulmonar Adultos Sociedad Chilena de Enfermedades Respiratorias

\section{Bibliografía}

1.- JORNADAS DE INVIERNO 2007. Sociedad Chilena de Enfermedades Respiratorias. "Función Pulmonar en adulto y niños. Lo que Ud. Debe saber". http://www. saval.cl/link.cgi/EducacionMedica/CursosyCongresos/ 2007_serjinv/

2.- MILLER M R, CRAPO R, HANKINSON J, BRUSASCO V, BURGOS F, CASABURI R, et al; ATS/ ERS Task Force. Standardisation of lung function testing: a) General considerations for lung function testing. Eur Respir J 2005; 26: 153-61. b) Standardisation of spirometry. Eur Respir J 2005; 26: 319-38.

3.- Global Strategy for the Diagnosis, Management, and Prevention of Chronic Obstructive Pulmonary Disease (GOLD). Updated 2005. http://www.goldcopd.dk/index uk.htm

4.- GUTIÉRREZ M, BEROÍZA T, BORZONE G, CAVIEDES I, CÉSPEDES J, GUTIÉRREZ M, et al. Espirometría: Manual de procedimientos. Sociedad Chilena de Enfermedades Respiratorias, 2006. Rev Chil Enf Respir 2007; 23: 31-42. 
5.- CRAPO R O. The role of reference values in interpreting lung function tests. Eur Respir J 2004 ; 24: 341-2.

6.- HANKINSON J L, ODENCRANTZ J R, FEDAN K B. Spirometric reference values from a sample of the general U.S. population. Am J Respir Crit Care Med 1999; 159: 179-87.

7.- KNUDSON R J, LEBOWITZ M D, HOLBERG C J, BURROWS B. Changes in the normal maximal expiratory flow-volume curve with growth and aging. Am Rev Respir Dis 1983; 127: 725-34.

8.- CORRALES R, FIERRO A M, GUTIÉRREZ M, LEIVA A, LINDERMAN C, MYER G, et al. Valores espirométricos normales para niños chilenos. Rev Chil Enf Respir 1992; 8: 148-57.

9.- MARAMBIO J A, SANDOVAL H, VALENZUELA P, MORENO R, DONOSO H, CONTRERAS G, et al. Espirometría en población general del Gran Santiago: Comparación con los valores de referencia de Knudson. Rev Chil Enf Respir. 1992; 8: 241.

10.- GUTIÉRREZ M, RIOSECO F, ROJAS A, CASANOVA D. Determinación de valores espirométricos en una población chilena normal mayor de 5 años, a nivel del mar. Rev Méd Chile 1996; 124: 1295-306.

11.- GUTIÉRREZ M, RIOSECO F, ROJAS A, CASANOVA D. Ecuaciones de referencia espirométricas en población chilena. Rev Chil Enf Respir. 1997; 13: 165-77.

12.- DÍAZ O, VILLAFRANCA C, LEIVA A, LISBOA C. Evaluación clínico funcional en la EPOC de acuerdo a Knudson y Gutiérrez. Rev Chil Enf Respir 1998; 14: 216.

13.- PALACIOS S, MARTÍNEZ A, FLORES G, PÉREZ G, OLMOS A. Comparación de valores de referencia foráneos y nacionales en el análisis de índices espirométricos en pacientes con estenosis mitral cerrada. Rev Chil Enf Respir 1998; 14: 215.

14.- ROJAS N, PLATERO A, QUIJADA D, STEVENS J, DUSSAUBAT N, OYARZÚN M. Comparación de valores espirométricos de referencia en una muestra de estudiantes de medicina. Rev Chil Enf Respir 2004; 20: 241 .

15.- GUTIÉRREZ M. Comparación de valores espirométricos chilenos con valores de referencia para población mejicano-americana de Estados Unidos. Rev Chil Enf Respir 2001; 17: 267. 